\title{
Novel Hendra Virus Variant Detected by Sentinel Surveillance of Horses in Australia
}

\author{
Edward J. Annand, ${ }^{1}$ Bethany A. Horsburgh, ${ }^{1}$ Kai Xu, Peter A. Reid, Ben Poole, \\ Maximillian C. de Kantzow, Nicole Brown, Alison Tweedie, Michelle Michie, John D. Grewar, \\ Anne E. Jackson, Nagendrakumar B. Singanallur, Karren M. Plain, Karan Kim, Mary Tachedjian, \\ Brenda van der Heide, Sandra Crameri, David T. Williams, Cristy Secombe, Eric D. Laing, Spencer Sterling, \\ Lianying Yan, Louise Jackson, Cheryl Jones, Raina K. Plowright, Alison J. Peel, Andrew C. Breed, \\ Ibrahim Diallo, Navneet K. Dhand, Philip N. Britton, Christopher C. Broder, Ina Smith, ${ }^{2}$ John-Sebastian Eden ${ }^{2}$
}

We identified and isolated a novel Hendra virus (HeV) variant not detected by routine testing from a horse in Queensland, Australia, that died from acute illness with signs consistent with HeV infection. Using whole-genome sequencing and phylogenetic analysis, we determined the variant had $\approx 83 \%$ nt identity with prototypic HeV. In silico and in vitro comparisons of the receptor-binding protein with prototypic $\mathrm{HeV}$ support that the human monoclonal antibody m102.4 used for postexposure prophylaxis and current equine vaccine will be effective against this variant.

$\mathrm{H}$ ighly pathogenic zoonotic Hendra virus (HeV) and Nipah virus (NiV) are prototypic members of the genus Henipavirus, family Paramyxoviridae, that have natural reservoirs in pteropodid flying foxes (1). These viruses exhibit wide mammalian host tropism,

Author affiliations: EquiEpiVet, Equine Veterinary and One Health Epidemiology, Aireys Inlet, Victoria, Australia (E.J. Annand); Department of Agriculture, Water, and the Environment Epidemiology and One Health Section, Canberra (E.J. Annand, M.C. de Kantzow, A.C. Breed); University of Sydney School of Veterinary Science and Institute for Infectious Diseases, Sydney, New South Wales, Australia (E.J. Annand, N. Brown, A. Tweedie, A.E. Jackson, K.M. Plain, N.K. Dhand); CSIRO Health and Biosecurity Black Mountain Laboratories, Canberra, Australian Capital Territory, Australia (E.J. Annand, M. Michie, I. Smith); Westmead Institute for Medical Research, Sydney (B.A. Horsburgh, K. Kim, J.-S. Eden); University of Sydney School of Medicine, Sydney (B.A. Horsburgh, C. Jones, P.N. Britton, J.-S. Eden); Ohio State University College of Veterinary Medicine, Columbus, Ohio, USA (K. Xu); Private equine veterinary practice, Brisbane, Queensland, Australia (P.A. Reid); Cooroora Veterinary Clinic, Cooroy, Queensland, Australia (B. Poole); JData, Cape Town, South Africa (J.D. Grewar); University of Pretoria, Pretoria, South Africa (J.D. Grewar); CSIRO Australian Centre for Disease Preparedness,
An updated quantitative PCR developed for routine surveillance resulted in subsequent case detection. Genetic sequence consistency with virus detected in grey-headed flying foxes suggests the variant circulates at least among this species. Studies are needed to determine infection kinetics, pathogenicity, reservoir-species associations, viral-host coevolution, and spillover dynamics for this virus. Surveillance and biosecurity practices should be updated to acknowledge $\mathrm{HeV}$ spillover risk across all regions frequented by flying foxes.

cause severe acute respiratory and encephalitic disease mediated by endothelial vasculitis, have high case-fatality rates, and cause chronic encephalitis among survivors (2-4). By March 2021, a total of 63 natural HeV spillovers had been recognized in horses in Australia,

Geelong, Victoria, Australia (N.B. Singanallur, M. Tachedjian, B. van der Heide, S. Crameri, D.T. Williams); Murdoch University School of Veterinary Medicine and The Animal Hospital, Murdoch, Western Australia, Australia (C. Secombe); Uniformed Services University of the Health Sciences Microbiology and Immunology, Bethesda, Maryland, USA (E.D. Laing, S. Sterling, L. Yan, C.C. Broder); Queensland Department of Agriculture and Fisheries Biosecurity Sciences Laboratory, Brisbane (L. Jackson, I. Diallo); Children's Hospital at Westmead, Infectious Diseases, Sydney (C. Jones, P.N. Britton); Montana State University, Bozeman, Montana, USA (R.K. Plowright); Griffith University Centre for Planetary Health and Food Security, Brisbane (A.J. Peel); University of Queensland School of Veterinary Science, Gatton, Queensland, Australia (A.C. Breed)

DOI: https://doi.org/10.3201/eid2803.211245

\footnotetext{
${ }^{1}$ These authors contributed equally to this article.

${ }^{2}$ These senior authors contributed equally to this article.
} 
resulting in 105 horse deaths $(5,6)$ and 4 deaths among 7 confirmed human cases (7). In southern Asia, NiV has caused zoonotic outbreaks with 70\%-91\% casefatality rates, resulting in $>700$ human deaths $(8-10)$. In response to the fatal disease threat posed by henipaviruses to humans and domestic animals, vaccines and postexposure prophylaxis (PEP) have been developed (11). A subunit vaccine, Equivac $\mathrm{HeV}$ (Zoetis, https:/ / www.zoetis.com.au), based on the soluble recombinant G-attachment glycoprotein (receptor-binding protein [RBP]) of $\mathrm{HeV}$ (HeV-sG), that has been used for horses in Australia since 2012 (12). The human monoclonal antibody (mAb) m102.4 has been administered as emergency PEP in 16 human cases and has demonstrated safety, tolerability, intended pharmacokinetics, and no immunogenicity in a phase 1 trial (13). Combinations of cross-reactive humanized fusion $(\mathrm{F})$ protein and RBP mABS have also been described for clinical development as PEP (14-16), and a human vaccine using $\mathrm{HeV}-\mathrm{sG}$ is now in phase 1 clinical trials (17).

Horses are the predominant species known to be infected with $\mathrm{HeV}$ by natural spillover from flying foxes; 2 canine (18) and all known human infections having resulted from close contact with infected horses. HeV transmission from Pteropus spp. (flying foxes) to horses is thought to occur primarily through contaminated urine (19). The spatial distribution of previously detected spillovers to horses and molecular $\mathrm{HeV}$ testing of flying fox urine suggested that transmission was predominantly from black flying foxes (BFF; $P$. alecto) and spectacled flying foxes (SFF; $P$. conspicillatus) $(19,20)$. However, serologic testing has detected antibodies to $\mathrm{HeV}$ or related henipaviruses among all 4 flying fox species in Australia (20-23). Of note, seroprevalence of IgG targeting the $\mathrm{HeV}$ RBP has been reported in $43 \%$ of grey-headed flying foxes (GHFF; P. poliocephalus) in South Australia and Victoria (22) and 60\% (169/284) in southeastern Queensland (21).

Australia hosts $>1$ million horses. Their grazing behavior, large respiratory tidal volume, and extensive highly vascularized upper respiratory epithelium may contribute to their vulnerability for $\mathrm{HeV}$ spillover (23). Detecting spillover to horses relies on attending veterinarians recognizing clinical manifestations consistent with $\mathrm{HeV}$ disease, sampling appropriately, and submitting samples for priority state laboratory testing (24). Passive surveillance using suspected disease testing is affected by a strong regional bias for areas where $\mathrm{HeV}$ has previously been detected and where domestic horse populations overlap with BFF distribution ranges, from eastern coastal Queensland to northern New South Wales (25). Testing for $\mathrm{HeV}$ is less commonly performed on horses with similar disease manifestations farther south in Australia because of a perception that spillover infection is less likely to occur in regions without BFF (26). Among $>1,000$ horses with manifestations consistent with $\mathrm{HeV}$ disease tested annually across regions of established risk, $<1 \%$ are found to be positive $(25,27)$.

Routine testing for equine $\mathrm{HeV}$ infection as part of priority disease investigation is specific for the matrix (M) gene (28). Additional nucleoprotein $(\mathrm{N})$ gene-specific testing (29) is limited to $\mathrm{HeV}$-positive samples that undergo confirmatory testing (30) or in the minority $(<7 \%$ nationally) of suspected equine $\mathrm{HeV}$ cases submitted directly to the national reference laboratory from states where spillover is considered less likely (25) and state testing is unavailable. This distinction is notable because it means that most horse-disease cases found negative for $\mathrm{HeV}$ are not investigated further, despite evidence that other viruses with potential spillover risk to horses, including novel related batborne paramyxoviruses, circulate in Australia (27,31-35). Likewise, animal health surveillance worldwide prioritizes targeted testing to exclude pathogens of established importance over open-ended diagnostic approaches, which are inherently more challenging to put in place and interpret.

Employing a transdisciplinary, interagency approach combining clinical-syndromic analysis and molecular and serologic testing, we explored the hypothesis that some severe viral disease-like manifestations in horses that are consistent with $\mathrm{HeV}$, despite the horse testing negative, could be caused by undetected spillover of novel paramyxoviruses from flying foxes that potentially pose similar zoonotic risk. Here we report the identification of a previously unrecognized variant of $\mathrm{HeV}(\mathrm{HeV}$-var), circulating as a second genotype lineage (HeV-g2), clinically indistinguishable from prototypic $\mathrm{HeV}$ infection, that resulted in severe neurologic and respiratory disease in a horse.

\section{Materials and Methods}

\section{Study Cohort}

A biobank of diagnostic specimens collected in Queensland during 2015-2018 was developed from horses that underwent quantitative reverse transcription PCR (RT-PCR) testing but were negative for $\mathrm{HeV}$ (28). We recorded clinical, epidemiologic, and sample-related data, including vaccination status and perceived exposure to flying foxes (inconsistently reported by submitting veterinarians). All samples were archived at $-80^{\circ} \mathrm{C}$. We applied a decision algorithm based on systematic interpretation of pathologic basis and syndromic analysis of clinical disease 
descriptions to categorize each case by likelihood of infectious viral cause (Appendix Table, https:/ /wwwnc.cdc.gov/EID/article/28/3/21-1245-App1.pdf). We plated samples (EDTA blood, serum, nasal swab, rectal swab) from cases assigned priority category 1 or 2 status, considered as having the highest likelihood of infectious cause, for serologic screening and high-throughput nucleic acid extraction using the MagMAX mirVANA and CORE pathogen kits (ThermoFisher, https:/ / www.thermofisher.com).

\section{Pan-paramyxovirus RT-PCR Screening}

We prepared cDNA from extracted RNA using Invitrogen SuperScript IV VILO Master Mix with ezDNase (ThermoFisher). A nested RT-PCR assay targeting the paramyxovirus L protein gene was adapted using primers developed elsewhere (36) and an AllTaq PCR Core kit (QIAGEN, https://www.qiagen.com). We identified amplicons corresponding to the expected size (584 $\mathrm{bp}$ ) by gel electrophoresis before purification with AMPure XP (Beckman Coulter, https://www.beckmancoulter.com). To capture any weak detections, we also prepared pools by equal-volume mixing all PCR products across plated rows. We performed next-generation sequencing using an Illumina iSeq with the Nextera XT DNA library preparation kit (Illumina, https://www. illumina.com). For analysis, we assembled reads with MEGAHIT (37) before identifying them by comparison to GenBank entries using BLAST (38).

\section{HeV-var Whole-Genome Sequencing}

We subjected samples positive for $\mathrm{HeV}$-var by paramyxovirus RT-PCR to meta-transcriptomic sequencing to determine the complete genome sequence and identify any co-infecting agents. RNA was reverse transcribed with Invitrogen SSIV VILO Master Mix (ThermoFisher) and FastSelect reagent (QIAGEN). We performed second-strand synthesis with Sequenase 2.0 (ThermoFisher) before DNA library preparation with Nextera XT (Illumina) and unique dual indexes. We performed sequencing on an Illumina NextSeq system to generate 100 million paired reads (75 bp) per library.

\section{Assembly and Comparative Genomic and Phylogenetic Analyses}

For genome assembly, we trimmed RNA sequencing reads and mapped them to a horse reference genome (GenBank GCA_002863925.1) using STAR aligner to remove host sequences. We assembled nonhost reads de novo with MEGAHIT (37) and compared them with the GenBank nucleotide and protein databases using blastn and blastx (38). We extracted the putative virus contig and remapped reads to this draft genome using bbmap version 37.98 (https:/ / sourceforge.net/projects/bbmap) to examine sequence coverage and identify misaligned reads. We extracted, aligned, and annotated the majority consensus sequence by reference to the prototype $\mathrm{HeV}$ strain using Geneious Prime version 2021.1.1 (https://www. geneious.com) and submitted it to GenBank (accession no. MZ318101).

For classification, we aligned the paramyxovirus polymerase (L) protein sequence according to International Committee on Taxonomy of Viruses (ICTV) guidelines (39). We prepared alignments of partial nucleocapsid $(\mathrm{N})$ and phosphoprotein $(\mathrm{P})$ nucleotide sequences with known $\mathrm{HeV}$ strains from the GenBank database. Phylogenies were prepared using a maximum likelihood approach in MEGA X (https:/ / www.megasoftware.net) according to the best-fit substitution model and 500 bootstrap replicates.

\section{Quantitative RT-PCR Development}

We adapted quantitative RT-PCR targeting the $\mathrm{HeV}$ $\mathrm{M}$ gene (28) to target $\mathrm{HeV}$-var. The duplex assay used the Applied Biosystems AgPath-ID One-Step RT-PCR kit (ThermoFisher), and distinguishes prototype and variant $\mathrm{HeV}$ strains. In brief, we combined $4 \mu \mathrm{L}$ RNA with $10 \mu \mathrm{L} 2 \times$ RT-PCR buffer, $0.8 \mu \mathrm{L} 25 \times$ RT-PCR enzyme mix, $2 \mu \mathrm{L}$ nuclease-free water, and $3.2 \mu \mathrm{L}$ primer/ probe mix $(0.6 \mu \mathrm{L}$ each primer, $0.3 \mu \mathrm{L}$ each probe from $10 \mu \mathrm{mol}$ stock; Table 1). We generated the reaction using $10 \mathrm{~min}$ at $50^{\circ} \mathrm{C}$ for cDNA synthesis, $10 \mathrm{~min}$ at $95^{\circ} \mathrm{C}$ for RT inactivation, and 50 cycles of $95^{\circ} \mathrm{C}$ for $15 \mathrm{~s}$ and $60^{\circ} \mathrm{C}$ for $30 \mathrm{~s}$ with FAM and HEX channels captured at the end of each cycle. As positive control, we synthesized

Table 1. Oligonucleotides used for duplex quantitative reverse transcription PCR targeting the matrix gene of novel Hendra virus variant from horse in Australia

\begin{tabular}{llcc}
\hline Virus & Name & Sequence, $5^{\prime} \rightarrow 3^{\prime *}$ & Reference \\
\hline Prototype & Mr_fwd_1 & CTTCGACAAAGACGGACCAA & $(34)$ \\
& Mr_rev_1 & CCAGCTCGTCGGACAAAATT & \\
\hline Variant & Mr_prb_1 & FAM-TGGCATCTT-ZEN-TCATGCTCCATCTCGG-IABk & Referent \\
& Mv_fwd_1 & TCTCGACAAGGACGGAGCTAA & \\
& Mv_rev_1 & CCGGCTCGTCGAACAAAATT & \\
\hline
\end{tabular}

${ }^{*}$ FAM and HEX 5' reporter dyes were combined with ZEN Internal Quencher and the 3' quencher lowa Black, and supplied by Integrated DNA Technologies (https://www.idtdna.com). 
gene fragments encoding a T7 promoter upstream of the partial $\mathrm{M}$ gene for both prototypic and variant $\mathrm{HeV}$ (Appendix Figure 1). We expressed RNA transcripts using the NEB HiScribe T7 High Yield RNA Synthesis kit (New England Biolabs, https://www.neb.com).

\section{Virus Isolation, Confirmation, and Neutralization Studies}

We attempted isolations in Vero cells (ATCC CCL-81) and primary kidney cells derived from black flying foxes (40). We confirmed them by cytopathogenic effect formation, quantitative RT-PCR, RNA sequencing, electron microscopy, and viral neutralization studies using $\mathrm{HeV}$ and isolated $\mathrm{HeV}$-var mAb m102.4 (Appendix).

\section{Serologic Analysis}

We performed serologic analysis using multiplex microsphere immunoassays with a Luminex MAGPIX system (https://www.luminexcorp.com). We performed initial screening for IgG using an extensive panel of bacterial (Leptospira, Brucella) and viral antigens (paramyxovirus, filovirus, coronavirus, flavivirus, alphavirus) coupled to MagPlex beads (Bio-Rad, https://www.bio-rad.com) for multiplex screening. We added blood or serum diluted 1:100 to the beads, with binding detected following the addition of a combination of biotinylated-protein-G and -A and streptavidin-R-phycoerythrin. We read median fluorescence intensity on the MAGPIX system (Luminex) targeting 100 beads per antigen and used a Bayesian latent class model to assess test performance and determine appropriate cutoffs for positive test classification (32). We also applied an IgM assay in which biotinylated equine IgM was used in place of biotinylated proteins A and G.

\section{In Silico Analysis of the RBP Homology and $\mathrm{mAb}$ Binding}

We compared the translated protein sequence of the HeV-var RBP sequence with established x-ray crystallography-derived structures of the HeV RBP protein bound to mAb m102.4 (41) and to ephrinB2 using SWISS-MODEL (https://swissmodel. expasy.org). We used the results to assess the ability of m102.4 to neutralize this variant and further establish the likelihood of antibodies produced by immunization with the $\mathrm{HeV}$ vaccine being protective against this variant.

\section{Results}

\section{Case Report}

In September 2015, veterinary care was sought for a 12-year-old Arabian gelding in southeastern
Queensland for severe disease consistent with $\mathrm{HeV}$ infection. The horse had always resided on the same property. Disease onset was acute; rapid deterioration occurred over 24 hours. Clinical assessment determined depressed (obtunded) demeanor, darkened red-to-purple change of the gingival mucous membranes with darker periapical line and prolonged capillary refill time, tachycardia (heart rate 75 beats/ $\mathrm{min})$, tachypnoea (60 breaths/min), normal rectal temperature $\left(38.0^{\circ} \mathrm{C}\right)$, muscle fasciculations, head pressing, and collapse.

$\mathrm{HeV}$ infection was suspected by the attending veterinarian, who had previously managed a confirmed case, on the basis of consistency with clinical disease manifestations and perception of plausible flying fox exposure. A nearby roost was known to host BFFs, GHFFs, and little red flying foxes (LRFF) of population sizes that varied seasonally and annually (42). Because of its moribund condition, the horse was humanely killed. We obtained postmortem nasal, oral, and rectal swab samples and combined them in $50 \mathrm{~mL}$ of sterile saline; we collected blood in an EDTA tube. Pooled swabs and blood samples were submitted to the Queensland Biosecurity Sciences Laboratory (Coopers Plains, Queensland, Australia) for priority $\mathrm{HeV}$ testing. Quantitative RT-PCR testing targeting the M gene did not detect viral RNA and ELISA testing did not detect HeV RBP IgG $(28,43)$.

\section{Identification of Novel HeV-var}

Given the high assigned likelihood of a zoonotic infectious cause (Appendix Table), we screened both the EDTA blood and pooled swab samples using panparamyxovirus RT-PCR (36). This identified the partial polymerase sequence of a novel paramyxovirus, most closely related to $\mathrm{HeV}(\approx 89 \%$ nt identity). Deep sequencing (mean coverage depth: $46.9 \times$ ) of blood RNA generated the near-full-length genome of a novel $\mathrm{HeV}$ (Figure 1, panel A). The virus was less abundant in the pooled swab sample; mean coverage depth was $0.6 \times$ reads, spanning only $9.9 \%$ of the genome (Figure 1, panel B). No other viruses were present in either sample, and other microbial reads assembled were from common microflora, including Staphylococcus aureus and Aeromonas, Veillonella, Pseudarthrobacter, Streptococcus, Acinetobacter, and Psychrobacter spp.

\section{Confirmation of HeV Infection}

A comparison of the primer and probe sequences used for the routine diagnostic PCR $(28,29)$ revealed multiple mismatches in the binding sites, explaining the failure of routine surveillance to 
detect this variant (Figure 2). A quantitative RTPCR assay was designed to detect both prototype and variant $\mathrm{HeV}$ strains in duplex (Table 1; Appendix Figures 1,2), which amplified the templates of each virus with similarly high efficiency (>94\%) and sensitivity, capable of detecting $<100$ copies of target RNA (Appendix Figure 2). The assay quantified results from the EDTA blood and pooled swabs samples, confirming RNA sequencing; the virus was more abundant in the EDTA blood (quantification cycle 26.87) than in the pooled swab samples (quantification cycle 30.67). We rescreened the priority cohort (864 samples from 350 cases in Queensland) using this novel assay but identified no additional cases. We successfully isolated virus from the EDTA blood sample of the case-animal in Vero cells. Electron microscopy of infected cells revealed cytoplasmic inclusion bodies (nucleocapsid aggregations; Figure 3, panel A) and enveloped viral-particle budding (Figure 3, panel B), consistent with $\mathrm{HeV}$ (Figure 3, panels A-D) (44).

Blood was tested for IgM and IgG against a panel of 33 antigens representative of bacterial and viral zoonoses $(32,45)$, including RBPs of paramyxoviruses: $\mathrm{HeV}, \mathrm{NiV}$, Cedar henipavirus (CedV), Mojiang henipavirus (MojV), Ghana bat henipavirus (GhV), Menangle, Grove, and Yeppoon and pararubula viruses. We observed no notable reactions for this animal-case blood in either the IgG or IgM assays, indicating a lack of detectable antibodies consistent with acute viremia.

\section{Genomic Analysis of Novel HeV-var}

We performed phylogenetic analyses of the novel $\mathrm{HeV}$-var with other known paramyxoviruses (Figure 4, panels A-C). Comparison of the nucleotide similarity of the novel $\mathrm{HeV}$-var to the $\mathrm{HeV}$ prototype strain (GenBank accession no. NC_001906) revealed an $83.5 \%$ pairwise identity across the genome (Figure 4, panel D). The L protein phylogeny revealed that the branch lengths of prototype and variant $\mathrm{HeV}$ to their common node did not exceed 0.03 substitutions/site (Figure 4, panels A, B). Therefore, the viruses were considered to be of the same species according to ICTV criteria (39). However, this HeV-var is clearly well outside known $\mathrm{HeV}$ diversity (Figure 4, panel C).

After this finding, comparison with a partial novel henipavirus $M$ gene sequence derived from a GHFF from South Australia in 2013 (46) revealed 99\% similarity to this $\mathrm{HeV}$-var. This, along with additional subsequent flying fox detections (47), suggests that this $\mathrm{HeV}$-var represents a previously undescribed lineage (HeV-g2), with reservoir-host infection across at least the range of this flying fox species.

\section{Analysis of the RBP}

Genomic sequencing showed greatest variability in the noncoding regions with mean pairwise genome identity higher $(86.9 \%)$ across coding regions (Figure 4, panel D). At the protein level, this $\mathrm{HeV}$-var shared $82.3 \%-95.7 \%$ (mean $92.5 \%$ ) aa identity to the $\mathrm{HeV}$ prototype (Table 2). Of note, the HeV-var RBP shared $92.7 \%$ aa identity with prototypic $\mathrm{HeV}$. Modeling of the novel HeV-var RBP structure based on the translated protein sequence using the $\mathrm{x}$-ray crystal structure of the prototypic HeV RBP published elsewhere (40) supports that the epitopes for binding ephrin-B2 receptor and $\mathrm{mAb}$ m102.4 remain functionally unchanged because of consistency between key residues (Figure 5). Indeed, mAb m102.4 neutralization assays revealed equivalent neutralization potency of m102.4 $(2.3 \mu \mathrm{g} / \mathrm{mL}$ of $\mathrm{m} 102.4$ neutralized 30 median tissue culture infectious dose of $\mathrm{HeV}$-var and $4.6 \mu \mathrm{g} / \mathrm{mL}$ of m102.4 neutralized 300 median tissue culture infectious dose of $\mathrm{HeV})$.

\section{Discussion}

We describe use of an innovative, syndromic riskbased targeted active sentinel surveillance activity for diagnostic investigation, extending from routine priority disease investigations, to identify a consequential virus. Based on ICTV criteria (39), this $\mathrm{HeV}$-var is a novel genotypic variant of $\mathrm{HeV}$, not a new Henipavirus species, but it evaded detection by routine diagnostic testing for $\mathrm{HeV}$ because of genomic divergence. Our findings highlight the potential of sentinel surveillance through One Health interagency and transdisciplinary syndromic infectious disease research to improve detection of emerging pathogens. We also describe a new assay for laboratory diagnosis and surveillance of this virus among humans and animals.

Comparing the translated amino acid sequences of this $\mathrm{HeV}$-var and prototypic $\mathrm{HeV}$ RBP in silico revealed no change in the mAb m102.4 or ephrin-B2 entry receptor binding sites. Similarly, we confirmed equivalent m102.4 neutralization in vitro for this $\mathrm{HeV}$ var and $\mathrm{HeV}$. As such, it is expected that current PEP using mAb m102.4 will also be effective against this $\mathrm{HeV}$-var. We emphasize that although the HeV RBP shares only $79 \%$ aa identity with NiV RBP, the HeV-sG subunit vaccine provided $100 \%$ protection against lethal challenge with both $\mathrm{HeV}$ and $\mathrm{NiV}$ in animal models (11). The high similarity between this HeV-var and $\mathrm{HeV}$ RBP (92.5\% aa identity), structural consistency of 


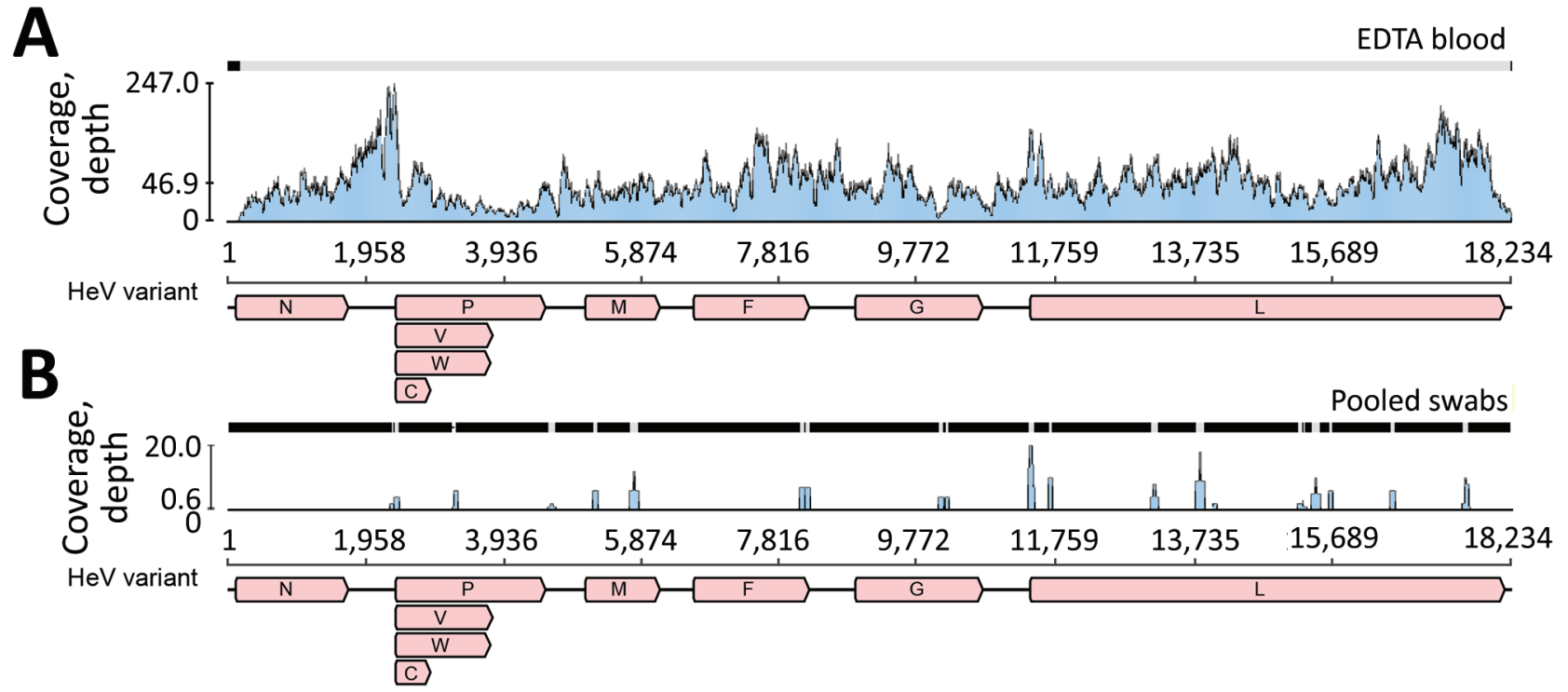

Figure 1. Sequence coverage of novel HeV variant from horse in Australia. The RNA sequencing reads were mapped to the novel HeV variant genome to examine coverage across the genome and depth for EDTA blood (A) and pooled swab samples (B). The x-axis shows the genome position with genes annotated and the $y$-axis shows the sequence read coverage (depth). Mean coverage depths were 46.9 for EDTA blood and 0.6 for pooled swab samples. V, W, and C indicate variably transcribed nonstructural proteins. F, fusion; G, glycoprotein; HeV, Hendra virus; M, matrix protein; N, nucleoprotein; P, phosphoprotein.

critical epitopes, and equivalent in vivo viral neutralization assays also support that current vaccination using the HEV RBP will elicit similarly protective antibodies against $\mathrm{HeV}$-var.

The $99 \%$ similarity between $\mathrm{HeV}$-var and a partial M-gene sequence detected in a GHFF from Adelaide in 2013 highlights that a greater diversity of $\mathrm{HeV}$ strains than previously recognized circulates among flying fox species in Australia and that this novel variant likely circulates as a relatively consistent sublineage (HeV-g2), at least across the range of GHFF. Subsequent identification of $\mathrm{HeV}-\mathrm{g} 2$ in GHFF and LRFF from regions without previous molecular $\mathrm{HeV}$ detection further support this understanding (47).

Our findings indicate the urgent need for prompt reassessment of $\mathrm{HeV}$ spillover risk for horses and handlers living in southern New South Wales, Victoria, and South Australia, where risk for $\mathrm{HeV}$ infection has been perceived as substantially lower than that in regions within the range of BFF distribution. Our findings indicate a need to update current molecular assays, which are not expected to distinguish between $\mathrm{HeV}$ and $\mathrm{HeV}$-var (HeV-g2), and increase surveillance testing in horses and screening of flying foxes for $\mathrm{HeV}-\mathrm{g} 2$ in these areas. These might further resolve the previously reported anomaly of high seropositivity despite low $\mathrm{HeV}$ detection within these species reported elsewhere (20-22).

Despite relatively high genetic divergence, the phenotypic similarity of this variant to prototypic $\mathrm{HeV}$, combined with the observed consistency of disease manifestations in horses, suggests that the 2 strains have equivalent pathogenicity and spillover potential. Further characterization of $\mathrm{HeV}$ genomic diversity and any host-species associations will increase our understanding of transmission dynamics as well as virus-host coevolution features, such as possible codivergence or founder effects. Indeed, as climate change and anthropogenic habitat loss alter the extent and nature of interspecies interactions, BFFs have rapidly expanded their range

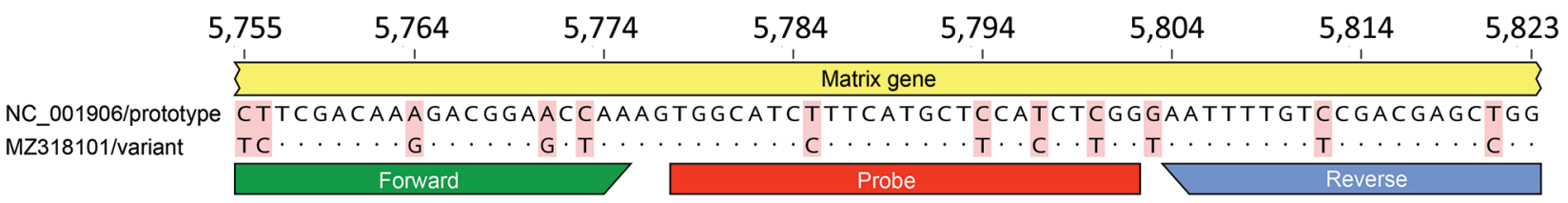

Figure 2. Genomic variation in the Hendra virus $(\mathrm{HeV})$ matrix gene assay primer/probe binding sites for novel HeV variant from horse in Australia. The genomic region targeted by the commonly used HeV matrix gene quantitative RT-PCR assay (28) was aligned and compared for the prototype and variant HeV strains. The genomic positions relative to the prototype strain (GenBank accession no. NC_001906) are shown at the top. Primers (forward and reverse) and probe binding sites are indicated by the colored bars. Mismatches between the sequences are highlighted with red shading; dots indicate identical bases. 

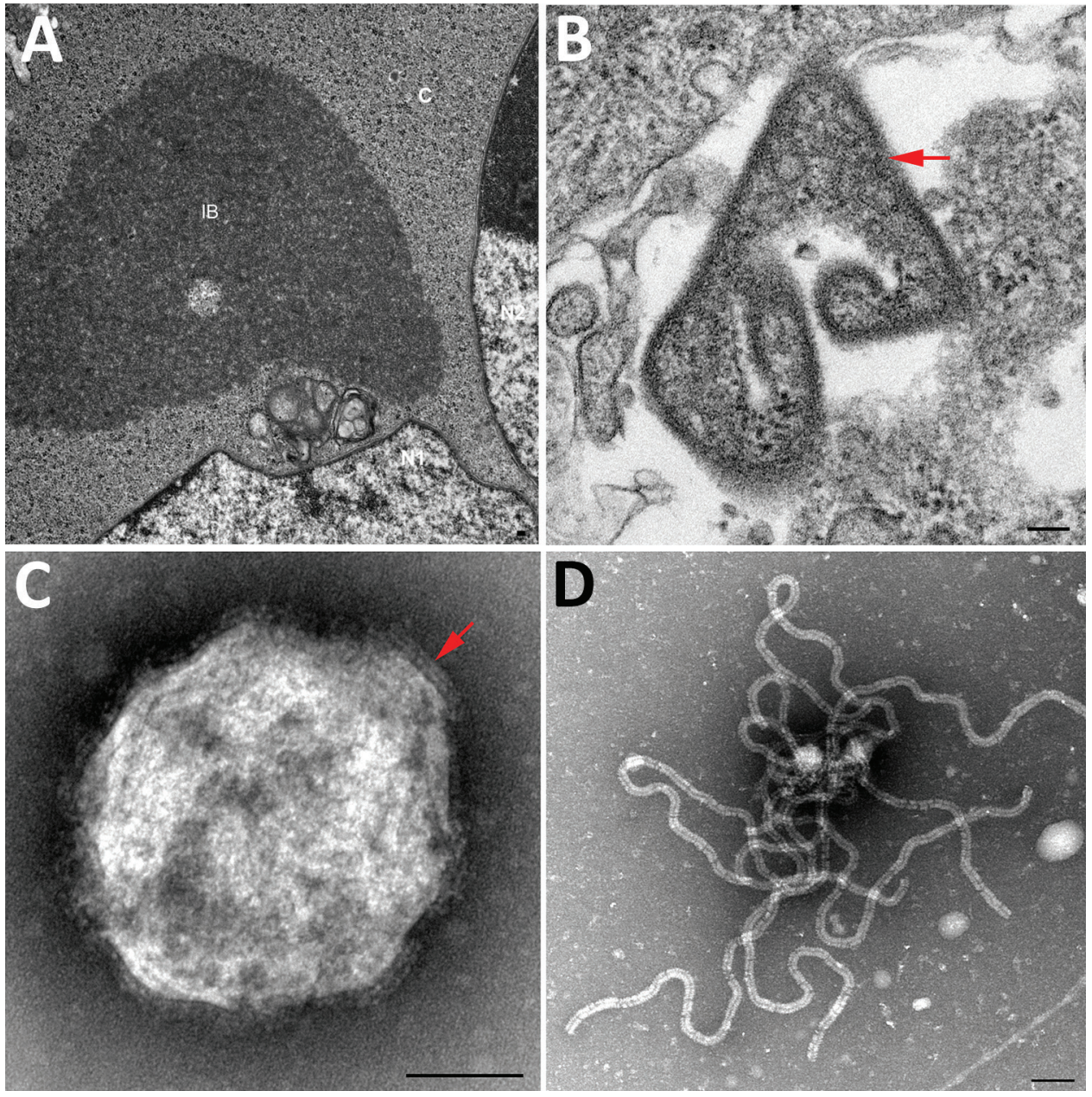

Figure 3. Transmission electron micrographs of Vero cells inoculated with the EDTA blood sample in study of novel Hendra virus variant from horse in Australia. A) Thin section showing inclusion body (IB) within the cytoplasm (C) of multinucleated (N1 and N2) syncytial cell. The nonmembrane bound IB consists of hollow nucleocapsids. B) Thin section showing virion (red arrow) with egress occurring at the plasma membrane. C) Negative contrast analysis shows a double-fringed envelope of the virion (red arrow). D) Negative contrast analysis shows strands of ribonucleic protein characteristic of the family Paramyxoviridae. Scale bars represent $100 \mathrm{~nm}$. southward, increasing their overlap with GHFFs (48). Sampling multiple species across time and space should inform how this variant strain circulates within and among flying fox species. Clearly, biosecurity practices should be updated to acknowledge spillover risk in all regions frequented by any species of flying fox.

Passive disease surveillance and biosecurity risk management for emerging diseases relies on recognition of suspected disease cases by clinical veterinarians, who play crucial roles relevant to animal and human health (24). Sporadic incidence of $\mathrm{HeV}$ and rare occurrence of Australian bat lyssavirus, yet high zoonotic consequence of both and lack of pathognomonic disease signs, inherently challenge surveillance of horses in Australia for these viruses. Critical and timely human postexposure management relies on confirmed animal-case diagnosis yet missed cases are inevitable, resulting in unmanaged risk of fatal zoonotic disease. Veterinarians are challenged in performing disease recognition by simultaneous obligations to serve both animals and animal owners, manage biosecurity risks, and meet Workplace Health and Safety Act and Biosecurity Act requirements $(24,49,50)$. Veterinary description of disease manifestations most consistent with $\mathrm{HeV}$ led us to prioritize this case in our research testing pathway. This research detection of $\mathrm{HeV}$-var highlights potential for improving emerging infectious disease surveillance through extending veterinarian-initiated risk-based suspect significant disease investigation, by selecting cases of highest likelihood of related viral cause and employing parallel serology and molecular testing pathways constructed to suit the available sample types and target diseases of highest clinical, species, and geographic relevance. These strategies build on the existing strength of systematic interpretation of clinical and field observations made by clinical veterinarians as part of existing submission and biosecurity protocols. This example serves as proof-of-concept for other disease contexts, highlighting the benefit of integrated transdisciplinary inquiry-based research approaches with routine biosecurity operations. Indeed, in 

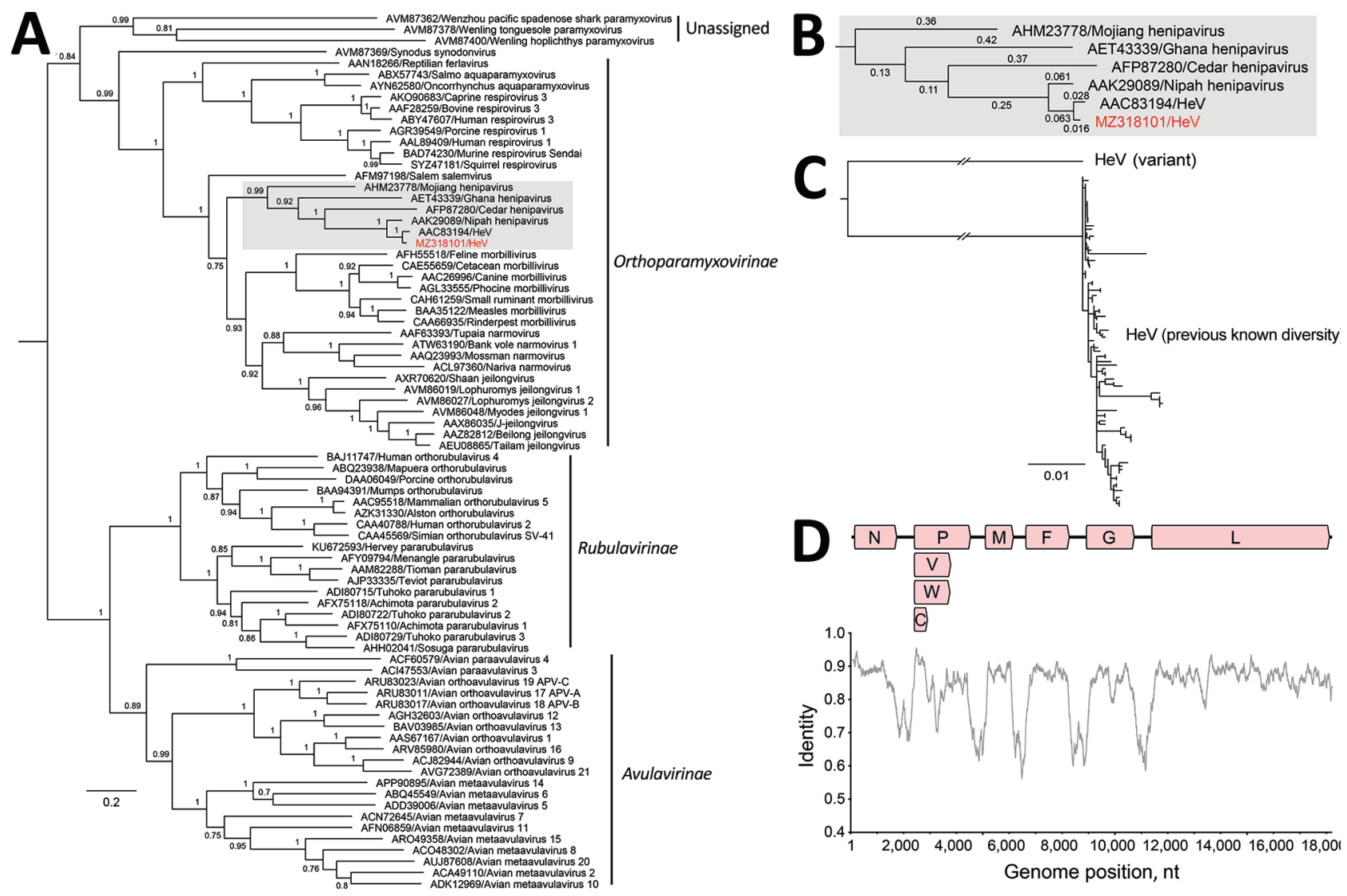

Figure 4. Phylogenomics of novel $\mathrm{HeV}$ variant from horse in Australia. A) Maximum-likelihood phylogeny of paramyxoviruses using complete $L$ protein sequences. Gray shading indicates henipaviruses, and red text indicates the novel HeV variant, which groups with the prototypic HeV. Bootstrap support values as proportions of 500 replicates are shown at nodes; values $<0.7$ are hidden. Scale bar indicates substitutions per site. B) Enlarged gray area from panel A shows branch lengths for the henipavirus clade. The branch leading back to the common ancestor of all known HeVs and the novel HeV variant does not exceed 0.03; thus, they are considered variants of the same species. C) Maximum-likelihood phylogeny of partial $\mathrm{N}$ and $\mathrm{P}$ where deep branch lengths have been collapsed for visualization only to demonstrate that the variant is well outside the known diversity of HeV. Scale bar indicates substitutions per site. D) Nucleotide genomic similarity of the variant compared with the prototypic HeV strain. V, W, and C indicate variably transcribed nonstructural proteins. F, fusion; G, glycoprotein; HeV, Hendra virus; L, paramyxovirus polymerase; M, matrix protein; N, nucleoprotein; $P$, phosphoprotein.

October 2021, a fatal horse-case of HeV-g2 infection near Newcastle, New South Wales, was detected through an updated quantitative RT-PCR incorporated into routine priority disease testing.

Acknowledging the limitations of this single case, which lacked tissue for histopathology and immunohistochemistry, it is nonetheless appropriate that this

\begin{tabular}{lcc}
\hline $\begin{array}{l}\text { Table 2. Protein lengths of novel Hendra virus variant from horse } \\
\text { in Australia and similarity to prototype strain* }\end{array}$ \\
\hline Protein & Length, aa & Similarity, \% \\
\hline Nucleoprotein & 532 & 96.6 \\
Phosphoprotein & 707 & 82.3 \\
Matrix & 352 & 95.7 \\
Fusion & 546 & 95.4 \\
Glycoprotein & 603 & 92.5 \\
Large & 2,244 & 95.7 \\
\hline
\end{tabular}

HeV-var (Hev-g2) be considered equally pathogenic to prototype $\mathrm{HeV}$ based on coherent and consistent clinical signs of disease and pathology, evidence of viraemia, the phylogenetic analysis indicating that the variant belongs to the $\mathrm{HeV}$ species, and the modeling of the interactions of the functional RBP domain to the virus entry ephrin-B2 receptor. Moreover, this case fits the case definition for $\mathrm{HeV}$ infection in Australia's AUSVETPLAN, which is that an animal tests positive to $\mathrm{HeV}$ using $\geq 1$ of $\mathrm{PCR}$, virus isolation, or immunohistochemistry (50).

Updated PCR diagnostics suitable for routine priority detection of this $\mathrm{HeV}$-var (Hev-g2) have been developed and are now used in many animal and human health laboratories in Australia. These findings demonstrate the limitation of exclusion-based testing 


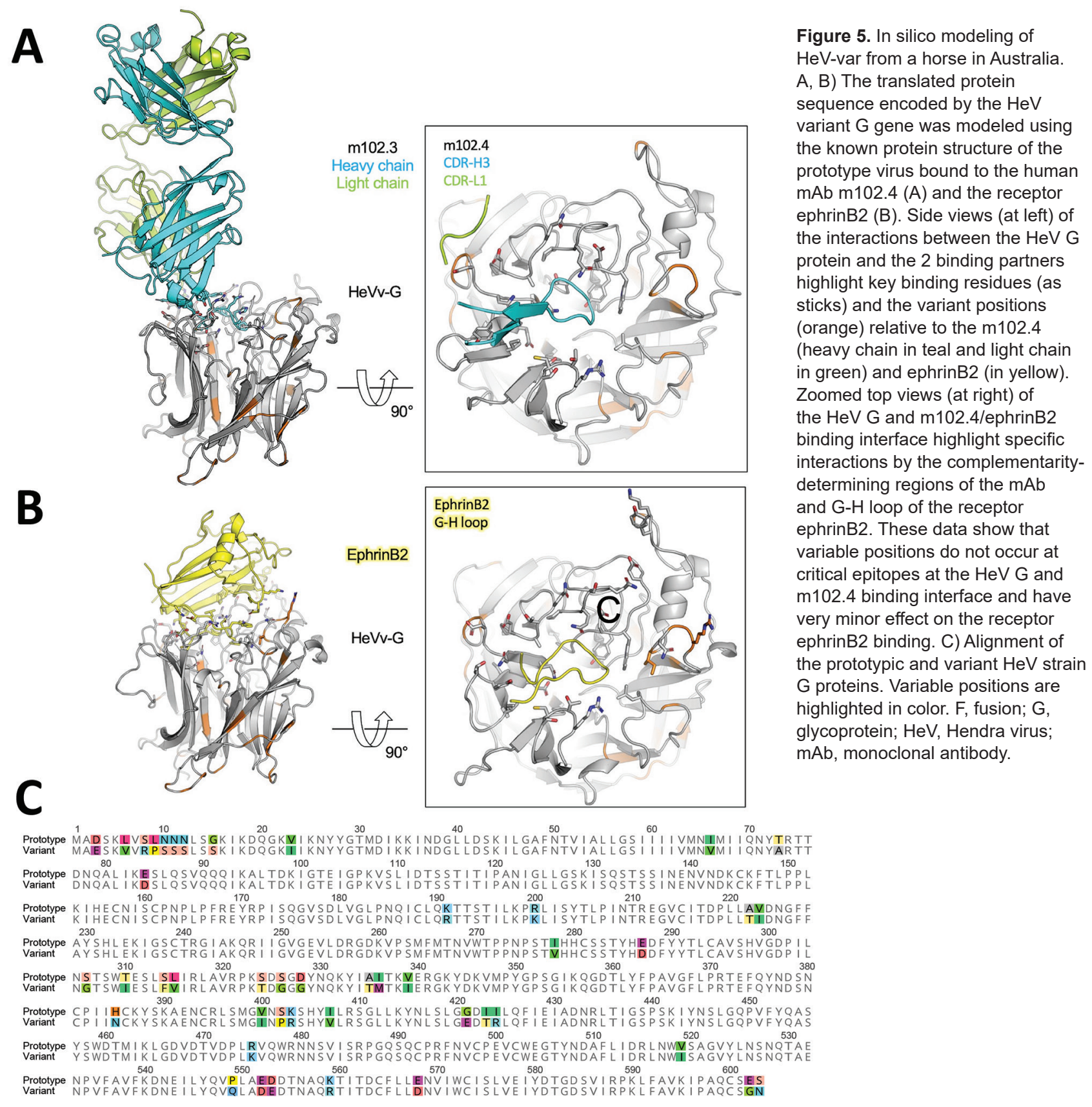

for emerging zoonoses and a gap in our understanding of how frequently detection of known zoonoses across a broad range of systems are missed because of the diagnostic tools used. Further investigations to determine the prevalence of $\mathrm{HeV}$-g2 circulation among and excretion from all flying fox species in Australia should be prioritized. The risk of zoonotic $\mathrm{HeV}$ disease in horses and human contacts should be interpreted across all regions frequented by all species of flying foxes, particularly those areas previously considered to be at low risk for $\mathrm{HeV}$ spillover.

\section{Acknowledgments}

The authors thank the staff of Biosecurity Sciences Laboratory, Brisbane, for processing samples and submitting information for this case and others in the comparative cohort. We thank Jennifer Barr for processing samples, Andrea Certoma and Mel Hargreaves for technical assistance in isolating the virus, and Jianning Wang for assisting with sequence comparisons. We thank David Bath, Robyn Martin, William Wong, and others from the Department of Agriculture Water and the Environment for supporting 
this project as part of the Biosecurity Innovation Program. We thank Jenny-Ann Toribio for PhD supervision. We thank Allan and Lyn Davies and the Dalara family foundation for their philanthropic financial support through the founding years of this research, without which this knowledge gap might not have been closed for much longer. We especially thank all veterinarians, owners, and caretakers who manage the health of horses and the public in relation to $\mathrm{HeV}$.

Funding was provided by the Australian Government Department of Agriculture, Water, and the Environment Biosecurity Innovation Program 2020-21 Project ID 202043, Metagenomic Investigation of Horses as Sentinels research; Dalara Foundation, philanthropic donation for: Horses and Human Health; University of Sydney, Sydney Institute for Infectious Diseases: internal seed funding; CSIRO Health and Biosecurity: internal funding; Australian Government Research Training Program scholarship; C.C.B. was supported by grant AI142764, National Institute of Allergy and Infectious Diseases, National Institutes of Health; AJP was supported by an ARC DECRA fellowship (DE190100710).

C.C.B. is a United States federal employee and inventor on US and foreign patents pertaining to soluble forms of Hendra virus and Nipah virus G glycoproteins and monoclonal antibodies against Hendra and Nipah viruses, whose assignees are the US Department of Health and Human Services (Washington, DC, USA) and Henry M. Jackson Foundation for the Advancement of Military Medicine Inc. (Bethesda, Maryland, USA). Remaining authors declare no competing interests. Opinions or assertions contained herein are the private ones of the author(s) and are not to be construed as official or reflecting the views of any of the Australia or international affiliated government or research agencies or official policy or position of the Uniformed Services University, US Department of Defense, or Henry M. Jackson Foundation for the Advancement of Military Medicine, Inc.

\section{About the Author}

Dr. Annand is an equine veterinarian epidemiologist in private practice and a research associate at the University of Sydney, School of Veterinary Science and Sydney Institute for Infectious Diseases. His core research interests include One Health infectious disease surveillance, and culturally conscious biosecurity risk management. Dr. Horsburgh is an early-career researcher at the Westmead Institute for Medical Research. She is interested in using single-copy assays and high-throughput sequencing to characterize viral genomes and understand their effect on human health.

\section{References}

1. Eaton BT, Broder CC, Middleton D, Wang L-F. Hendra and Nipah viruses: different and dangerous. Nat Rev Microbiol. 2006;4:23-35. https://doi.org/10.1038/nrmicro1323

2. Selvey LA, Wells RM, McCormack JG, Ansford AJ, Murray K, Rogers RJ, et al. Infection of humans and horses by a newly described morbillivirus. Med J Aust. 1995;162:642-5. https://doi.org/10.5694/j.1326-5377.1995. tb126050.x

3. Wong KT, Tan CT. Clinical and pathological manifestations of human henipavirus infection. Curr Top Microbiol Immunol. 2012;359:95-104. https:// doi.org/10.1007/ 82_2012_205

4. Playford EG, McCall B, Smith G, Slinko V, Allen G, Smith I, et al. Human Hendra virus encephalitis associated with equine outbreak, Australia, 2008. Emerg Infect Dis. 2010;16:219-23. https://doi.org/10.3201/eid1602.090552

5. Murray K, Rogers R, Selvey L, Selleck P, Hyatt A, Gould A, et al. A novel morbillivirus pneumonia of horses and its transmission to humans. Emerg Infect Dis. 1995; 1:31-3. https://doi.org/10.3201/eid0101.950107

6. Business Queensland. Summary of Hendra virus incidents in horses. 2015 [cited 2020 Feb 14]. https:/ / www.business. qld.gov.au/industries/service-industries-professionals/ service-industries/veterinary-surgeons/guidelines-hendra/ incident-summary

7. New South Wales Health. Summary of human cases of Hendra virus infection [cited 2021 Apr 23]. https:/ / www. health.nsw.gov.au/Infectious/controlguideline/Pages/ hendra-case-summary.aspx

8. Arunkumar G, Chandni R, Mourya DT, Singh SK, Sadanandan R, Sudan P, et al.; Nipah Investigators People and Health Study Group. Outbreak investigation of Nipah virus disease in Kerala, India, 2018. J Infect Dis. 2019;219:1867-78. https://doi.org/10.1093/infdis/jiy612

9. Ching PK, de los Reyes VC, Sucaldito MN, Tayag E, Columna-Vingno AB, Malbas FF Jr, et al. Outbreak of henipavirus infection, Philippines, 2014. Emerg Infect Dis. 2015;21:328-31. https:// doi.org/10.3201/eid2102.141433

10. Nikolay B, Salje H, Hossain MJ, Khan AKMD, Sazzad HMS, Rahman M, et al. Transmission of Nipah virus -14 years of investigations in Bangladesh. N Engl J Med. 2019;380:180414. https:// doi.org/10.1056/NEJMoa1805376

11. Amaya $M$, Broder CC. Vaccines to emerging viruses: Nipah and Hendra. Annu Rev Virol. 2020;7:447-73. https://doi.org/10.1146/annurev-virology-021920-113833

12. Middleton D, Pallister J, Klein R, Feng YR, Haining J, Arkinstall R, et al. Hendra virus vaccine, a One Health approach to protecting horse, human, and environmental health. Emerg Infect Dis. 2014;20:372-9. https:/ / doi.org/ 10.3201/eid2003.131159

13. Playford EG, Munro T, Mahler SM, Elliott S, Gerometta M, Hoger KL, et al. Safety, tolerability, pharmacokinetics, and immunogenicity of a human monoclonal antibody targeting the G glycoprotein of henipaviruses in healthy adults: a first-in-human, randomised, controlled, phase 1 study. Lancet Infect Dis. 2020;20:445-54. https:/ / doi.org/10.1016/ S1473-3099(19)30634-6

14. Dong J, Cross RW, Doyle MP, Kose N, Mousa JJ, Annand EJ, et al. Potent henipavirus neutralization by antibodies recognizing diverse sites on Hendra and Nipah virus receptor binding protein. Cell. 2020;183:1536-50.e17. https:/ / doi.org/10.1016/j.cell.2020.11.023

15. Doyle MP, Kose N, Borisevich V, Binshtein E, Amaya M, Nagel M, et al. Cooperativity mediated by rationally selected combinations of human monoclonal antibodies target- 
ing the henipavirus receptor binding protein. Cell Rep. 2021;36:109628. https:/ / doi.org/10.1016/j.celrep.2021.109628

16. Dang HV, Cross RW, Borisevich V, Bornholdt ZA, West BR, Chan Y-P, et al. Broadly neutralizing antibody cocktails targeting Nipah virus and Hendra virus fusion glycoproteins. Nat Struct Mol Biol. 2021;28:426-34. https:/ / doi. org/10.1038/s41594-021-00584-8

17. Geisbert TW, Bobb K, Borisevich V, Geisbert JB, Agans KN, Cross RW, et al. A single dose investigational subunit vaccine for human use against Nipah virus and Hendra virus. NPJ Vaccines. 2021;6:23. https:/ / doi.org/10.1038/ s41541-021-00284-w

18. Kirkland PD, Gabor M, Poe I, Neale K, Chaffey K, Finlaison DS, et al. Hendra virus infection in dog, Australia, 2013. Emerg Infect Dis. 2015;21:2182-5. https://doi.org/ 10.3201/eid2112.151324

19. Edson D, Field H, McMichael L, Vidgen M, Goldspink L, Broos A, et al. Routes of Hendra virus excretion in naturally-infected flying-foxes: implications for viral transmission and spillover risk. PLoS One. 2015;10:e140670. https://doi.org/10.1371/journal.pone.0140670

20. Burroughs AL, Durr PA, Boyd V, Graham K, White JR, Todd S, et al. Hendra virus infection dynamics in the grey-headed flying fox (Pteropus poliocephalus) at the southern-most extent of its range: further evidence this species does not readily transmit the virus to horses. PLoS One. 2016;11:e0155252. https:// doi.org/10.1371/journal. pone. 0155252

21. Edson D, Peel AJ, Huth L, Mayer DG, Vidgen ME, McMichael L, et al. Time of year, age class and body condition predict Hendra virus infection in Australian black flying foxes (Pteropus alecto). Epidemiol Infect. 2019;147:e240. https:/ / doi.org/10.1017/S0950268819001237

22. Boardman WSJ, Baker ML, Boyd V, Crameri G, Peck GR, Reardon T, et al. Seroprevalence of three paramyxoviruses; Hendra virus, Tioman virus, Cedar virus and a rhabdovirus, Australian bat lyssavirus, in a range expanding fruit bat, the grey-headed flying fox (Pteropus poliocephalus). PLoS One. 2020;15:e0232339. https:/ / doi.org/10.1371/journal. pone. 0232339

23. Plowright RK, Eby P, Hudson PJ, Smith IL, Westcott D, Bryden WL, et al. Ecological dynamics of emerging bat virus spillover. Proc R Soc B. 2015;282:20142124.

24. Annand EJ, Reid PA, Johnson J, Gilbert GL, Taylor M, Walsh $\mathrm{M}$, et al. Citizens' juries give verdict on whether private practice veterinarians should attend unvaccinated Hendra virus suspect horses. Aust Vet J. 2020;98:273-9. https:/ / doi.org/10.1111/avj.12957

25. Animal Health Australia. Animal health surveillance quarterly [cited 2021 May 21]. file:/ / C:/Users/tkp3/ Downloads/Animal\%20Health $\% 20$ Surveillance $\% 20$ Quarterly\%20Vol\%2026\%20Iss\%201\%20\%20(2).pdf

26. Government of South Australia. Department of Primary Industries and Regions. Hendra virus in South Australia. 2018 [cited 2021 May 21]. https:/ / pir.sa.gov.au/biosecurity/ animal_health/horses/hendra_virus\#toc1

27. Agnihotri K, Pease B, Oakey J, Campbell G. Confirmation of Elsey virus infection in a Queensland horse with mild neurologic signs. J Vet Diagn Invest. 2016;28:445-8. https:// doi.org/10.1177/1040638716652652

28. Smith IL, Halpin K, Warrilow D, Smith GA. Development of a fluorogenic RT-PCR assay (TaqMan) for the detection of Hendra virus. J Virol Methods. 2001;98:33-40. https://doi.org/10.1016/S0166-0934(01)00354-8

29. Feldman KS, Foord A, Heine HG, Smith IL, Boyd V, Marsh GA, et al. Design and evaluation of consensus PCR assays for henipaviruses. J Virol Methods. 2009;161:52-7. https:/ / doi.org/10.1016/j.jviromet.2009.05.014

30. Yuen KY, Fraser NS, Henning J, Halpin K, Gibson JS, Betzien L, et al. Hendra virus: epidemiology dynamics in relation to climate change, diagnostic tests and control measures. One Health. 2021;12:100207. https:// doi.org/10.1016/j.onehlt.2020.100207

31. Annand EJ, Reid PA. Clinical review of two fatal equine cases of infection with the insectivorous bat strain of Australian bat lyssavirus. Aust Vet J. 2014;92:324-32. https://doi.org/10.1111/avj.12227

32. Annand E, Barr J, Singanallur Balasubramanian N, Reid P, Boyd V, Burneikienè-Petraitytė R, et al. Spillover of bat borne Rubulavirus in Australian horses - horses as sentinels for emerging infectious diseases. Int J Infect Dis. 2020;101:401-2. https://doi.org/10.1016/j.ijid.2020.09.1051

33. Barr J, Smith C, Smith I, de Jong C, Todd S, Melville D, et al. Isolation of multiple novel paramyxoviruses from pteropid bat urine. J Gen Virol. 2015;96:24-9. https:/ / doi.org/ 10.1099/vir.0.068106-0

34. Marsh GA, de Jong C, Barr JA, Tachedjian M, Smith C, Middleton D, et al. Cedar virus: a novel henipavirus isolated from Australian bats. PLoS Pathog. 2012;8:e1002836. https:/ / doi.org/10.1371/journal.ppat.1002836

35. Vidgen ME, de Jong C, Rose K, Hall J, Field HE, Smith CS. Novel paramyxoviruses in Australian flying-fox populations support host-virus co-evolution. J Gen Virol. 2015;96:161925. https://doi.org/10.1099/vir.0.000099

36. Tong S, Chern S-WW, Li Y, Pallansch MA, Anderson LJ. Sensitive and broadly reactive reverse transcription-PCR assays to detect novel paramyxoviruses. J Clin Microbiol. 2008;46:2652-8. https:/ / doi.org/10.1128/JCM.00192-08

37. Li D, Liu C-M, Luo R, Sadakane K, Lam T-W. MEGAHIT: an ultra-fast single-node solution for large and complex metagenomics assembly via succinct de Bruijn graph. Bioinformatics. 2015;31:1674-6. https://doi.org/10.1093/ bioinformatics/btv033

38. Altschul SF, Gish W, Miller W, Myers EW, Lipman DJ. Basic local alignment search tool. J Mol Biol. 1990;215:403-10. https://doi.org/10.1016/S0022-2836(05)80360-2

39. Rima B, Balkema-Buschmann A, Dundon WG, Duprex P, Easton A, Fouchier R, et al.; ICTV Report Consortium. ICTV virus taxonomy profile: Paramyxoviridae. J Gen Virol. 2019;100:1593-4. https:// doi.org/10.1099/jgv.0.001328

40. Crameri G, Todd S, Grimley S, McEachern JA, Marsh GA, Smith C, et al. Establishment, immortalisation and characterisation of pteropid bat cell lines. PLoS One. 2009;4:e8266. https:// doi.org/10.1371/journal.pone.0008266

41. Xu K, Rockx B, Xie Y, DeBuysscher BL, Fusco DL, Zhu Z, et al. Crystal structure of the Hendra virus attachment $G$ glycoprotein bound to a potent cross-reactive neutralizing human monoclonal antibody. PLoS Pathog. 2013;9:e1003684. https://doi.org/10.1371/journal.ppat.1003684

42. Australian Government Department of Agriculture, Water and the Environment. Monitoring flying-fox populations [cited 2021 May 28]. https:/ / www.environment.gov.au/ biodiversity/threatened/species/flying-fox-monitoring

43. Colling A, Lunt R, Bergfeld J, McNabb L, Halpin K, Juzva S, et al. A network approach for provisional assay recognition of a Hendra virus antibody ELISA: test validation with low sample numbers from infected horses. J Vet Diagn Invest. 2018;30:362-9. https:// doi.org/10.1177/1040638718760102

44. Hyatt AD, Zaki SR, Goldsmith CS, Wise TG, Hengstberger SG. Ultrastructure of Hendra virus and Nipah virus within cultured cells and host animals. Microbes Infect. 2001;3:297306. https:// doi.org/10.1016/S1286-4579(01)01383-1 
45. Laing E, Yan L, Sterling S, Broder C. A Luminex-based multiplex assay for the simultaneous detection of glycoprotein specific antibodies to ebolaviruses, marburgviruses, and henipaviruses. Int J Infect Dis. 2016;53:108-9. https://doi.org/10.1016/j.ijid.2016.11.272

46. Wang J, Anderson D, Valdeter S, Chen H, Walker S, Meehan B, et al. A novel henipavirus in bats, Australia. In: Proceedings of the One Health EcoHealth Congress. One Health EcoHealth, 4-7 Dec 2016; Melbourne, Australia [cited 2021 Feb 18]. https:/ / publications.csiro.au/ $\mathrm{rpr} /$ pub?pid=csiro:EP173003

47. Wang J, Anderson DE, Halpin K, Hong X, Chen H, Walker $\mathrm{S}$, et al. A new Hendra virus genotype found in Australian flying foxes. Virol J. 2021;18:197.

48. Roberts BJ, Catterall CP, Eby P, Kanowski J. Latitudinal range shifts in Australian flying-foxes: a re-evaluation.
Austral Ecol. 2012;37:12-22. https:/ / doi.org/10.1111/ j.1442-9993.2011.02243.x

49. Mendez DH, Judd J, Speare R. Unexpected result of Hendra virus outbreaks for veterinarians, Queensland, Australia. Emerg Infect Dis. 2012;18:83-5. https://doi.org/10.3201/ eid1801.111006

50. Animal Health Australia. Australia Veterinary Emergency Plan AUSVETPLAN, Response policy brief Hendra virus infection, version 4.0. Animal Health Australia; 2016 [cited 2021 May 5]. https:// animalhealthaustralia.com.au/ ausvetplan/download/5621

Address for correspondence: Edward Annand, Sydney School of Veterinary Science, University of Sydney, Camden, NSW 2570, Australia; email: ed.annand@sydney.edu.au

\section{November 2020}

\section{Respiratory Infections}

- The Problem of Microbial Dark Matter in Neonatal Sepsis

- Two Pandemics, One ChallengeLeveraging Molecular Test Capacity of Tuberculosis Laboratories for Rapid COVID-19 Case-Finding

- Measuring Timeliness of Outbreak Response in the World Heath Organization African Region, 2017-2019

- Challenges to Achieving Measles Elimination, Georgia, 2013-2018

- Phage-Mediated Immune Evasion and Transmission of Livestock-Associated Methicillin-Resistant Staphylococcus aureus in Humans

- Validated Methods for Removing Select Agent Samples from Biosafety Level 3 Laboratories

- Epidemiology of COVID-19 Outbreak on Cruise Ship Quarantined at Yokohama, Japan, February 2020

- Expert Taskforce for the COVID-19 Cruise Ship Outbreak

- Analysis of SARS-CoV-2 Transmission in Different Settings, Brunei

- Case-Control Study of Use of Persona Protective Measures and Risk for SARS-CoV-2 Infection, Thailand

- Transmission of SARS-CoV-2 During Long-Haul Flight

- Nowcasting (Short-Term Forecasting) of Influenza Epidemics in Local Settings, Sweden, 2008-2019 A. Spreco et al. 2670

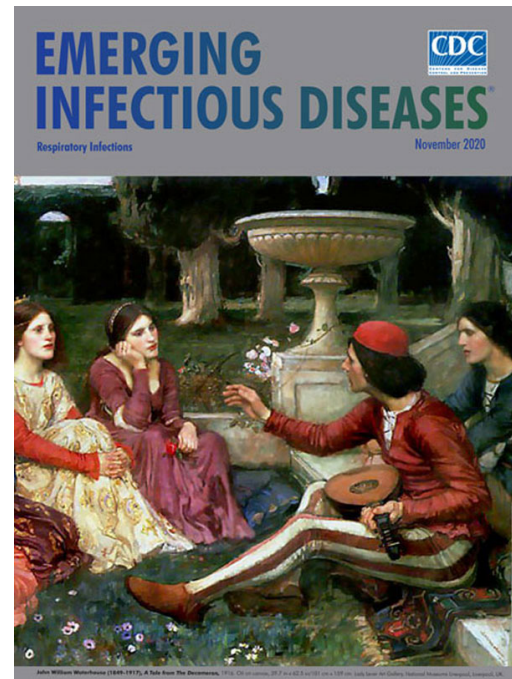

- High Dengue Burden and Circulation of 4 Virus Serotypes among Children with Undifferentiated Fever, Kenya, 2014-2017

- Endotheliopathy and Platelet Dysfunction as Hallmarks of Fatal Lassa Fever

- Systematic Review and Meta-Analyses of Incidence for Group B Streptococcus Disease in Infants and Antimicrobial Resistance, China

- Streptococcus pneumoniae Serotype 12F-CC4846 and Invasive Pneumococcal Disease after Introduction of 13-Valent Pneumococcal Conjugate Vaccine, Japan, 2015-2017
- Azithromycin to Prevent Pertussis in Household Contacts, Catalonia and Navarre, Spain, 2012-2013

- Modeling Treatment Strategies to Inform Yaws Eradication

- Multidrug-Resistant Candida auris Infections in Critically Ill Coronavirus Disease Patients, India, April-July 2020

- Potential Role of Social Distancing in Mitigating Spread of Coronavirus Disease, South Korea

- SARS-CoV-2 Virus Culture and Subgenomic RNA for Respiratory Specimens from Patients with Mild Coronavirus Disease

- Asymptomatic Transmission of SARS-CoV-2 on Evacuation Flight

- Worldwide Effects of Coronavirus Disease Pandemic on Tuberculosis Services, January-April 2020

- Preventing Vectorborne Transmission of Zika Virus Infection During Pregnancy, Puerto Rico, USA, 2016-2017

- Multidrug-Resistant Hypervirulent Group B Streptococcus in Neonatal Invasive Infections, France, 2007-2019

- Epileptic Seizure after Use of Moxifloxacin in Man with Legionella longbeachae Pneumonia

- Thresholds versus Anomaly Detection for Surveillance of Pneumonia and Influenza Mortality 